\title{
IMPROVING REGIONAL OVERPOWER PROTECTION TRIP SET POINT VIA CHANNEL OPTIMIZATION
}

\author{
DODDY KASTANYA \\ Reactor Physics Branch \\ Candu Energy Inc. \\ 2285 Speakman Drive, Mississauga, ON, Canada L5B 1K1 \\ ${ }^{*}$ Corresponding author. E-mail : Doddy.kastanya@candu.com \\ Received March 03, 2011 \\ Accepted for Publication November 23, 2011
}

In recent years, a new algorithm has been introduced to perform the regional overpower protection (ROP) detector layout optimization for CANDU ${ }^{\circledR}$ reactors. This algorithm is called DETPLASA. This algorithm has been shown to successfully come up with a detector layout which meets the target trip set point (TSP) value. Knowing that these ROP detectors are placed in a number of safety channels, one expects that there is an optimal placement of the candidate detectors into these channels. The objective of the present paper is to show that a slight improvement to the TSP value can be realized by optimizing the channelization of these ROP detectors. Depending on the size of the ROP system, based on numerical experiments performed in this study, the range of additional TSP improvement is from $0.16 \% \mathrm{FP}$ (full power) to $0.56 \% \mathrm{FP}$.

KEYWORDS : Regional Overpower Protection, CANDU, Channelization, Optimization, Simulated Annealing

\section{INTRODUCTION}

In the CANada Deuterium Uranium (CANDU ${ }^{\circledR 1}$ ) reactor design, the regional overpower protection (ROP) systems (which is also known as the neutron overpower protection or NOP $[1,2,3]$ ) protect the reactor against excessively high power in the fuel which could lead to dryout $^{2}$. The power increase could be caused by a localized power peaking within the core or a general increase in the core power level during a slow-loss-of-regulation (SLOR) event.

There are two ROP systems in the CANDU $600 \mathrm{MW}$ (CANDU 6) design. Each system consists of three independent safety channels. Each system includes a number of fast-responding, self-powered flux detectors which are distributed throughout the core within vertical and horizontal assemblies. The shutdown system number 1 (SDS1) is a rod-based shutdown system. It consists of cadmium shutoff rods (SOR) which are located at the top of the

\footnotetext{
${ }^{1}$ CANDU is a registered trademark of Atomic Energy of Canada Limited.

${ }^{2}$ Dryout is a condition where the fuel is operating at temperatures higher than the desired temperature. The coolant around the fuel sheath surface produces many small bubbles that could eventually coalesce into a vapour film enveloping the fuel element. This reduces the heat transfer from the fuel to the coolant and in turn further elevates the fuel temperature.
}

reactor. The rods are released into the core when this shutdown system is actuated. The SDS1 ROP system consists of 34 self-powered, platinum-clad, straight individually replaceable (SIR) detectors which are located in 16 out of 26 vertical assemblies. It should be noted that these vertical assemblies are shared with other flux detectors used for reactivity control and flux mapping. The shutdown system number 2 (SDS 2) is a liquid-poisonbased shutdown system. The SDS2 is connected to highly concentrated gadolinium solution which is stored in high pressure tanks. The gadolinium solution is released into the moderator when the SDS2 is actuated. The SDS2 ROP system includes 24 detectors of the same types as SDS1 which are located in 7 out of 9 horizontal assemblies. The three safety channels for SDS1 are designated as channels D, E, and F; while for SDS2 they are designated as channels $\mathrm{G}, \mathrm{H}$, and J. Fig. 1 shows a diagram of the shutdown systems in CANDU 6 reactor.

The placement of the ROP detectors into the vertical and horizontal assemblies for the SDS1 and SDS2, respectively, is a constrained discrete optimization problem. Recently, the DETPLASA algorithm has been developed to perform the optimization of the ROP detector placement [4]. The DETPLASA algorithm utilizes the simulated annealing (SA) stochastic optimization algorithm [5] to find a quasi optimal solution to the problem. Within the algorithm, the objective of the optimization is to maximize 


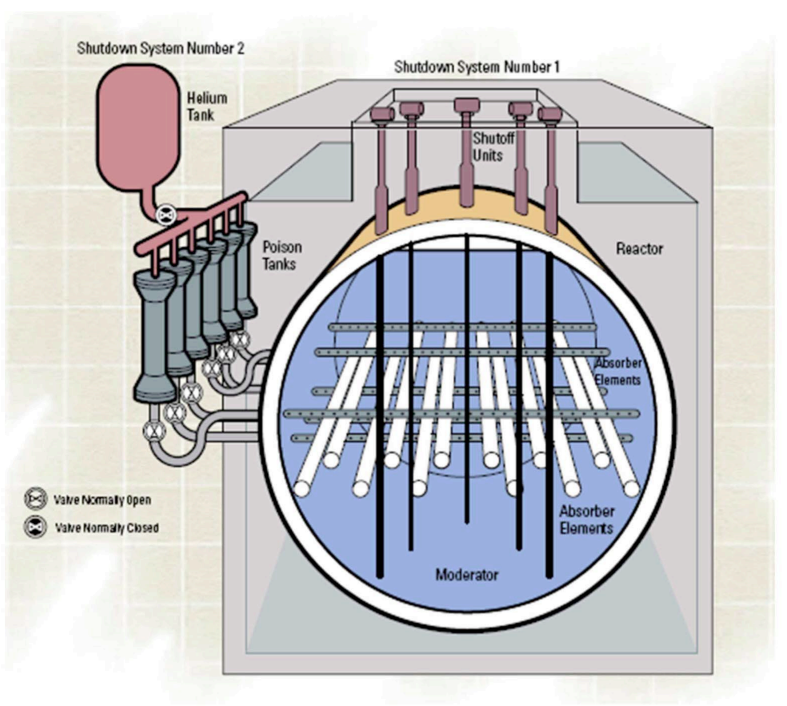

Fig. 1. CANDU 6 Shutdown Systems.

the trip set point (TSP) value ${ }^{3}$. This TSP value is calculated using a probabilistic method in the ROVER-F code [6]. The output from executing this algorithm is a detector layout which satisfies the target TSP value. These detectors are placed into the available safety channels; however, there is no guarantee that the placement of these detectors into these safety channels is optimized. Therefore, additional optimization on how the detectors are channelized can be performed. The objective of this particular optimization is to further increase the TSP value. This additional optimization is the subject presented in this paper.

This paper is structured as follows. In the next section, the concept of channelization is introduced. Brief overviews of how the TSP is calculated and how the detector layout optimization is performed are presented in Sections 3 and 4, respectively. Section 5 discusses the channelization optimization and Section 6 presents some results from various numerical experiments to test this methodology. Finally, the paper is closed with some conclusions.

\section{THE CONCEPT OF CHANNELIZATION}

The ROP detectors for each shutdown system are placed into a few safety channels. There are two main reasons for having multiple safety channels in the core. The first one is related to the safety aspect of the operation. It is desirable that there are multiple sets of detectors ${ }^{4}$ distributed throughout the core which continuously monitor the power changes in the core. The second reason is related to the economic aspect of the operation. Since the signal from the safety channel is responsible for actuating the shutdown system, it is desirable to have multiple safety channels so as to avoid actuating the shutdown system spuriously. It should be noted that signals from all safety channels are entered into a specific voting logic so that the eventual decision to actuate the shutdown system will be made after considering the inputs from all safety channels.

In the current CANDU 6 design, each shutdown system consists of three safety channels. For SDS1, there are 12,11 , and 11 detectors in safety channel D, E, and F, respectively. For SDS2, each safety channel has 8 detectors. For CANDU 6 analysis, the voting logic for actuating a shutdown system used in calculating the TSP value in the ROVER-F code is called the "worst-two-outof-two" voting logic. This voting logic assumes that the best safety channel to trip is unavailable and the remaining two safety channels trip. It should also be noted that a channel will trip when the detector reading of any detector within that channel surpasses its TSP value. How this voting logic is incorporated into the ROP TSP calculation is discussed in the next section.

\section{OVERVIEW OF THE ROP TSP CALCULATION}

The safety requirement of an ROP system is that it must actuate a reactor trip before the onset of intermittent dryout (OID) in any fuel channel. In practice, it is both physically and mechanically prohibitive to detect the dryout of a fuel bundle among 4560 fuel bundles in a 380-channel CANDU 6 reactor. Therefore, instead of directly monitoring the OID, the ROP analyses are performed by considering two quantities, namely the margin-to-dryout (MTD) and the margin-to-trip (MTT). The former is defined as the ratio between the channel power at which dryout will first occur (the corresponding channel power level is called the critical channel power or CCP) and the actual channel power (CP). The latter one is defined as the ratio between the reactor power at which the ROP system will actuate the shutdown system and the actual reactor power. The relation between these two quantities can be expressed by the following inequality

$$
M T T \leq M T D
$$

or,

$$
\frac{T S P}{\Phi} \leq \frac{C C P}{C P}
$$

where TSP is the trip set point, $\Phi$ is the detector reading,

\footnotetext{
${ }^{3}$ It should be noted that the TSP quoted corresponds to the lowest TSP value obtained after evaluating all core configurations considered in the analysis (these various core configurations are called Flux Shapes - see Section 3).

${ }^{4}$ Each of these sets is placed into a unique safety channel.
} 
$C C P$ is the critical channel power, and $C P$ is the channel power.

In the design and operation of the ROP system, a nominal flux distribution (and, hence, the power distribution) refers to normal operating core configuration where the average zone controller level is around $50 \%$, the adjusters are fully inserted, and the mechanical control absorber rods as well as the shutoff rods are fully withdrawn. This configuration is defined as the nominal case since this is expected to be a representative average over the life of the reactor. The departure in the neutron flux distribution from this nominal distribution can be categorized into two types:

a. Flux Shapes. The flux shapes are defined as various flux and power distributions caused by changing the device configuration (including zone-controller fills) or changes in the xenon distribution from the nominal distribution.

b. Ripples. Ripples are defined as variations in the core power distribution that are due to fuelling and changes in the burnup distribution. The ripples used in the ROP can be generated from core-follow simulations or come from operating data from the plant.

The basic ROP safety requirement can then be expanded using these two definitions. The requirement is that for any flux shape $k$ and ripple $q$, each safety channel must trip before the power in any fuel channel reaches the critical channel power for that fuel channel. This means that the detector locations, detector channelizations in the safety channels, and the trip set point (TSP) must be determined carefully such that for each flux shape considered, there is at least one detector $j_{p, i}$ in each safety channel $i$ which satisfies the following expression:

$$
T S P\left(j_{p, i}\right) \leq \Phi(j, k) \times r_{C P R L}(k, q)
$$

where $\operatorname{TSP}\left(j_{p, i}\right)$ is the installed trip set point for protecting detector $j$ (the subscript $p$ is used to emphasize that it is a protecting detector), in voting logic channel $i$; $\Phi(j, k)$ is the normalized ${ }^{5}$ detector reading at detector $j$ for flux shape $k$ (and may include several calibration terms depending on plant operation); and, $r_{C P R L}(k, q)$ is the minimum critical power ratio (i.e., the MTD) for flux shape $k$ and ripple $q$. This is the basic deterministic equation for determining the trip set point value.

Since each component involved in determining the TSP value has some uncertainties related to its value, then the deterministic approach for calculating the TSP value is no longer applicable. A probabilistic calculation is required. In order to determine the TSP value that satisfies the licensed trip probability (98\%/average), a probabilistic approach is utilized by the ROVER-F code which is used in the ROP TSP analysis for CANDU 6 reactors. The system trip probability value can be written as follows ${ }^{6}$ :

$$
P_{T}=1-\int_{0}^{\infty} Q_{C M}(x) P_{N T}(x) d x
$$

where $P_{T}$ is the trip probability, $Q_{C M}$ is the common-mode error distribution, $P_{N T}$ is the non-trip probability of the detector systems (accounting for all safety channels in the shutdown system and the unique trip voting logic used in the analysis), and $x$ is any possible value of limiting rippled critical power ratio. The trip set point (TSP) comes into Eq. (4) through the $P_{N T}$ term. The correct TSP value is determined iteratively where in each iteration the "guessed" TSP value has to be adjusted until the required trip probability $\left(P_{T}\right)$ is achieved. The details on how the probabilistic TSP calculation is done can be found in [6].

\section{OVERVIEW OF THE CURRENT METHODOLOGY FOR OPTIMIZING THE ROP DETECTOR LAYOUT CONFIGURATION}

The design process to determine the detector layout for the ROP system in a CANDU reactor can be divided into two major steps, namely: the deterministic design process and the stochastic optimization process. The deterministic design process can be further divided into two parts: margin optimization and logical (Boolean) reduction of potential detectors. Recently, a new algorithm, called DETPLASA, has been introduced to perform the stochastic optimization process. This new algorithm utilizes the simulated annealing technique as its optimization engine. Comprehensive descriptions of the detector layout optimization using the DETPLASA algorithm as well as using the earlier tool called $\mathrm{DLO}^{7}$ can be found in [4].

\section{METHODOLOGY FOR IMPROVING THE TSP VALUE FURTHER: OPTIMIZATION OF CHANNELIZATION PROCESS}

Regardless of which tool is employed (e.g., DETPLASA or DLO), at the end of a ROP detector design process, the designer will have a quasi optimized detector layout. The detectors have been placed into a number of safety channels. However, at this point the designer does not know whether or not the placement of detectors into the

\footnotetext{
${ }^{5}$ The detector reading is normalized to the detector reading for the nominal case and thus is invariant to ripple variations.

${ }^{6}$ It should be noted that the trip probability calculation is done for each flux shape $k$ in each SDS. However, for simplicity, the SDS and case (flux shape) indices are dropped from this equation.

${ }^{7}$ DLO stands for Detector Layout Optimization. This is one of the modules in the ROVER-F code.
} 
safety channel is the best. Additional optimization can be performed in trying to optimize the channelization of the selected detectors from the original optimization process. The methodology for doing this is described below.

The channelization optimization process begins with an ROP detector layout resulting from the design process where the detectors have already been placed in the safety channels. For the discussion in this paper, instead of using three-safety-channel system as in CANDU 6 design, it is assumed that there are four safety channels in the system (as proposed for the Advanced CANDU Reactor or ACR design). Related to this, the "worst-two-out-of-two" voting logic is no longer applicable. Another voting logic called "worst-two-out-of-four" is used. In this case, the best safety channel to trip is assumed unavailable and then the remaining three safety channels are evaluated using the "worst-two-out-of-two" voting logic. With the initial channelization from the detector layout optimization, the corresponding TSP value can be calculated and it will become the initial TSP value which will be improved by the additional optimization. For an ROP system which has 80 detectors, there are more than $2 \times 10^{45}$ possible channelizations $^{8}$ (assuming equal number of detectors in each safety channel). It is obvious that it is computationally prohibitive to evaluate all of these permutations. Therefore, the use of a stochastic optimization technique, such as the SA technique, is appropriate.

It should be noted that within the SA technique, the goodness of each detector layout configuration is measured by the objective function, $F_{o b j}$, which is defined as

$$
F_{o b j}=-\left(\frac{T S P_{C}}{T S P_{T}}\right)
$$

where $T S P_{C}$ and $T S P_{T}$ are the current and target trip set point, respectively. For each problem, the target TSP value is set such as some improvement over the initial TSP value is attained. It should also be noted that the TSP values are calculated using the ROVER-F code.

The basic implementation of the SA technique for the channelization optimization can be described as follows:

a. The first step is to initialize the unitless "temperature" parameter, $T$, to a high value (an analogy to the physical annealing process), initialize the counter for the number of histories (called ITER) for this particular temperature to 0 , initialize the counter for the number of temperatures (called TCOUNT) to 1, and find an initial solution (and subsequently save it as the best solution, i.e., $F_{o b j}^{m i n}$ ). b. Load the best solution (so far) as the current solution and increase ITER by one. c. Check if ITER is greater than ITER $_{\max }$ (the maximum number of histories allowed for a particular temperature). If it is true, then the temperature should be decreased (assuming that TCOUNT is still less than TCOUNT $_{\max }$ - the maximum number of temperature reductions; otherwise, the optimization process is done) using an appropriate cooling schedule ${ }^{9}$, initialize ITER to 1 , and then go to step " $d$ ". If it is not true, proceed directly to step "d".

d. Perform a perturbation to the current configuration. This is done by performing the following steps:

i. Select the first safety channel randomly and then select a detector within this safety channel randomly.

ii. Select the second safety channel which is different from the first safety channel and then randomly select a detector within this second safety channel. iii. Exchange the two detectors found in step ' $\mathrm{i}$ ' and 'ii'. e. After a new configuration (solution) is established, calculate the corresponding objective function (i.e., calculating the TSP value for the configuration).

f. Check if the new objective function is less than the previous objective function. If so, then check whether it is lower than the best (minimum) objective function. If it is lower than the best solution, the new solution will be saved as both the current solution and the best solution (and also update the $F_{o b j}^{\min }$ value); otherwise, the solution is only saved as the current solution. If the objective function is higher than the current objective function, then it can still be accepted (i.e., saved as the current solution for the next iteration) if it satisfies the following criterion

$$
\exp \left\{-\frac{\Delta F_{o b j}}{T}\right\} \geq R(0,1)
$$

where $R(0.1)$ is a random number between 0 and 1 (and is generated every time this evaluation is needed), $\Delta F_{o b j}$ represents the increase in the TSP value, and $T$ is the current temperature. Save the appropriate current solution and proceed to step "c".

The algorithm above shows that for a given "temperature" the detector configuration is varied in a Markov chain Monte Carlo based method based on what is commonly known as the Metropolis sampling algorithm. The minimum observed value of the objective function, corresponding to the highest TSP value, is tracked. It should be noted that considering the size of the problem, the result from the optimization problem will likely be a quasioptimal solution instead of the ultimate optimal solution. Finally, it should also be noted that the steps shown above

\footnotetext{
${ }^{8}$ The permutation for selecting the first 20 detectors from 80 detectors to be placed in the first safety channel is $\frac{80 !}{20 ! 6 !}$. The permutation for selecting the next 20 detectors from the remaining 60 detectors to be placed in the second safety channel is $\frac{60 !}{20 ! 40 !}$. The permutation for selecting the next 20 detectors from the remaining 40 detectors to be placed in the third (and, consequently, the fourth) safety channel is $\frac{40 !}{20 ! 20 !}$. Therefore, the overall number of permutations is $\frac{80 !}{(20 !)^{4}}$ or $2.04 \times 10^{45}$.

${ }^{9}$ Cooling schedule refers to the formulation used for reducing the SA temperature from one cycle to the next.
} 
are very similar to the steps in the DETPLASA algorithm, except for step "d". In DETPLASA, the perturbation is done on the sequence of flux shapes used for selecting the detectors followed by the actual detector selection.

\section{RESULTS AND DISCUSSIONS}

The channelization optimization has been performed on a number of ROP systems with varying sizes. There are six sizes examined, namely 56-detector, 60 -detector, 64-detector, 68-detector, 72-detector, and 76-detector configurations. There is nothing special about these particular sizes. These sizes were selected so that the detectors can be split evenly into four safety channels. For each configuration, 20 channelization optimization runs are executed in order to observe the average behaviour from these runs. Table 1 summarizes the results from these runs.

Presented in this table are the best TSP values from

Table 1. Results of Channelization Optimizations.

\begin{tabular}{|c|c|c|c|c|c|c|}
\hline \multirow{2}{*}{ Optimization Run } & \multicolumn{6}{|c|}{ Number of Detectors } \\
\hline & 56 & 60 & 64 & 68 & 72 & 76 \\
\hline 1 & $1.2319^{\mathrm{a}}$ & 1.2360 & 1.2363 & 1.2378 & 1.2397 & 1.2394 \\
\hline 2 & 1.2330 & 1.2389 & 1.2388 & 1.2401 & 1.2419 & 1.2422 \\
\hline 3 & 1.2331 & 1.2395 & 1.2387 & 1.2403 & 1.2428 & 1.2424 \\
\hline 4 & 1.2300 & 1.2391 & 1.2387 & 1.2393 & 1.2428 & 1.2418 \\
\hline 5 & 1.2332 & 1.2389 & 1.2370 & 1.2404 & 1.2424 & 1.2424 \\
\hline 6 & 1.2335 & 1.2380 & 1.2385 & 1.2391 & 1.2429 & 1.2429 \\
\hline 7 & 1.2335 & 1.2282 & 1.2393 & 1.2388 & 1.2426 & 1.2418 \\
\hline 8 & 1.2335 & 1.2368 & 1.2374 & 1.2386 & 1.2424 & 1.2426 \\
\hline 9 & 1.2320 & 1.2351 & 1.2389 & 1.2401 & 1.2429 & 1.2424 \\
\hline 10 & 1.2333 & 1.2382 & 1.2395 & 1.2394 & 1.2424 & 1.2411 \\
\hline 11 & 1.2281 & 1.2343 & 1.2385 & 1.2410 & 1.2419 & 1.2413 \\
\hline 12 & 1.2329 & 1.2397 & 1.2394 & 1.2404 & 1.2424 & 1.2424 \\
\hline 13 & 1.2333 & 1.2393 & 1.2385 & 1.2400 & 1.2426 & 1.2420 \\
\hline 14 & 1.2335 & 1.2389 & 1.2387 & 1.2389 & 1.2426 & 1.2395 \\
\hline 15 & 1.2335 & 1.2392 & 1.2383 & 1.2406 & 1.2429 & 1.2423 \\
\hline 16 & 1.2335 & 1.2392 & 1.2399 & 1.2388 & 1.2416 & 1.2429 \\
\hline 17 & 1.2332 & 1.2380 & 1.2392 & 1.2403 & 1.2426 & 1.2316 \\
\hline 18 & 1.2333 & 1.2389 & 1.2388 & 1.2404 & 1.2425 & 1.2428 \\
\hline 19 & 1.2335 & 1.2379 & 1.2391 & 1.2391 & 1.2424 & 1.2423 \\
\hline 20 & 1.2335 & 1.2394 & 1.2385 & 1.2383 & 1.2419 & 1.2428 \\
\hline Average TSP & 1.2328 & 1.2377 & 1.2386 & 1.2396 & 1.2423 & 1.2414 \\
\hline Maximum TSP & 1.2335 & 1.2395 & 1.2395 & 1.2404 & 1.2429 & 1.2429 \\
\hline Initial TSP & 1.2319 & 1.2366 & 1.2373 & 1.2386 & 1.2373 & 1.2394 \\
\hline Average Improvement & $0.09 \% \mathrm{FP}^{\mathrm{b}}$ & $0.11 \% \mathrm{FP}$ & $0.13 \% \mathrm{FP}$ & $0.10 \% \mathrm{FP}$ & $0.50 \% \mathrm{FP}$ & $0.20 \% \mathrm{FP}$ \\
\hline Maximum Improvement & $0.16 \% \mathrm{FP}$ & $0.29 \% \mathrm{FP}$ & $0.22 \% \mathrm{FP}$ & $0.18 \% \mathrm{FP}$ & $0.56 \% \mathrm{FP}$ & $0.35 \% \mathrm{FP}$ \\
\hline
\end{tabular}

${ }^{a} 1.2319$ corresponds to $123.19 \% \mathrm{FP}$.

${ }^{\mathrm{b}} \mathrm{FP}$ - Full Power. 


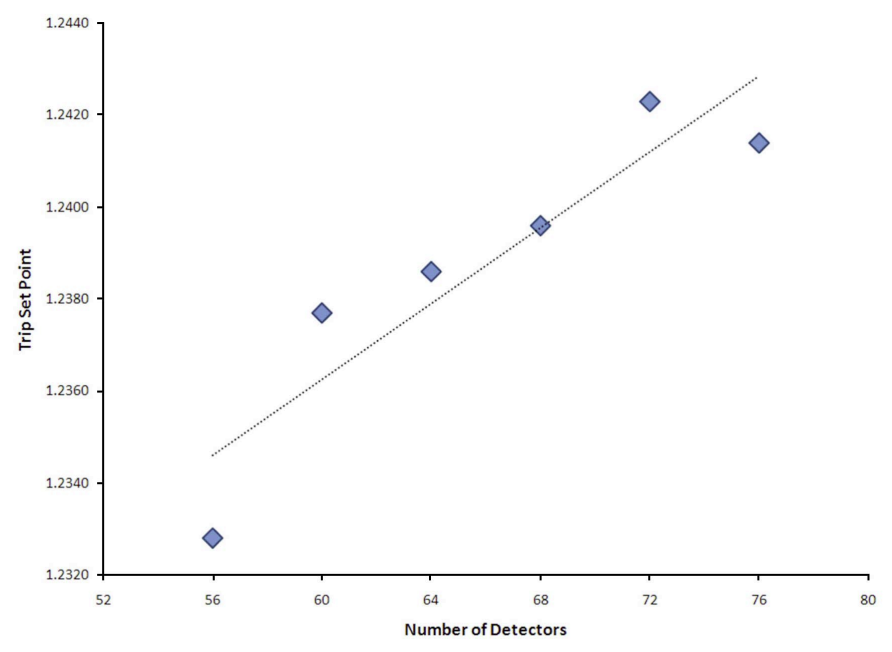

Fig. 2. Average Trip Set Point (TSP) as a Function of Number of Detectors.

individual optimization runs. The initial TSP value for each configuration is also presented. Having calculated the average TSP value and determined the maximum TSP value from the 20 optimization runs for each configuration, one can determine the average improvement as well as the maximum improvement in the TSP value. The values are also summarized in the table. These results show that from this numerical experiment, the maximum improvement in the TSP value ranges from $0.16 \% \mathrm{FP}^{10}$ to $0.56 \% \mathrm{FP}$.

Fig. 2 shows the behaviour of the TSP value as a function of number of detectors in the system. Each TSP value in this figure is an average over the 20 optimization runs. This figure depicts an expected behaviour where the TSP increases as more and more detectors are added into the system. However, it should be noted that this behaviour does not continue indefinitely. A "saturation" region will eventually be reached where adding detectors into the system does not provide further improvement to the TSP value.

Up to this point, it has been shown that the additional channelization optimization can provide additional improvement to the TSP value. In the remaining of this section, we will try to understand why such improvement can be attained.

In Section 2, it is mentioned that it is desirable to have the detector spread out throughout the core. This principle to some extent is also applicable to each safety channel in the system. It is desirable to have detectors within one safety channel be spread out somewhat evenly throughout the core. In order to quantify how well the detector are distributed, a new quantity called index of distribution $\left(I_{D i s t}\right)$ is introduced. First of all, the core is split into four quadrants, namely Q1, Q2, Q3, and Q4.

\footnotetext{
${ }^{10} \mathrm{FP}-$ Full Power.
}

The index of distribution is defined as

$$
I_{D \text { bंt }}=\frac{1 \times N_{Q 1}+2 \times N_{Q 2}+3 \times N_{Q 3}+4 \times N_{Q 4}}{10}
$$

where $N_{Q i}$ is the number of detectors placed in quadrant " $i$ ". Needless to say, this is just an example of weighting schemes that can be used for defining $I_{D i s t}$.

Using Eq. (7), one can calculate the index of distributions for various designs. Tables 2 and 3 summarize the index of distribution for 72-detector and 76-detector configurations, respectively. In each table, the initial values of $I_{D i s t}$ as well as the values correspond to the optimized configuration are presented. For each configuration, there is an ideal distribution of detectors within each safety channel. For example, for the 72-detector configuration, there are 18 detectors in each safety channel. So, the ideal configuration involves two quadrants with 5 detectors and the other two quadrants with 4 detectors. There are six possible combinations to determine which quadrant has 5 or 4 detectors. Using Eq. (7), one can calculate the range of ideal $I_{D i s t}$ values based on these six combinations. The range is between 4.3 and 4.7. A similar calculation for the 76-detector configuration gives a range between 4.6 and 4.9 .

Fig. 3 presents the results graphically. From this figure and Tables 2 and 3, one can observe that for both configurations, the optimized solution gives a smaller range of $I_{D i s t}$ which is also closer to the ideal range. This is an indication that the detectors are better distributed in the core. Furthermore, it should be highlighted that for the 72-detector configuration, three out of the four safety channels have the $I_{D i s t}$ values within the ideal range.

Finally, it should be mentioned that since the original optimization run using the DETPLASA algorithm has evaluated a significant number of detector layout configurations, one would expect that, by chance, the channels 


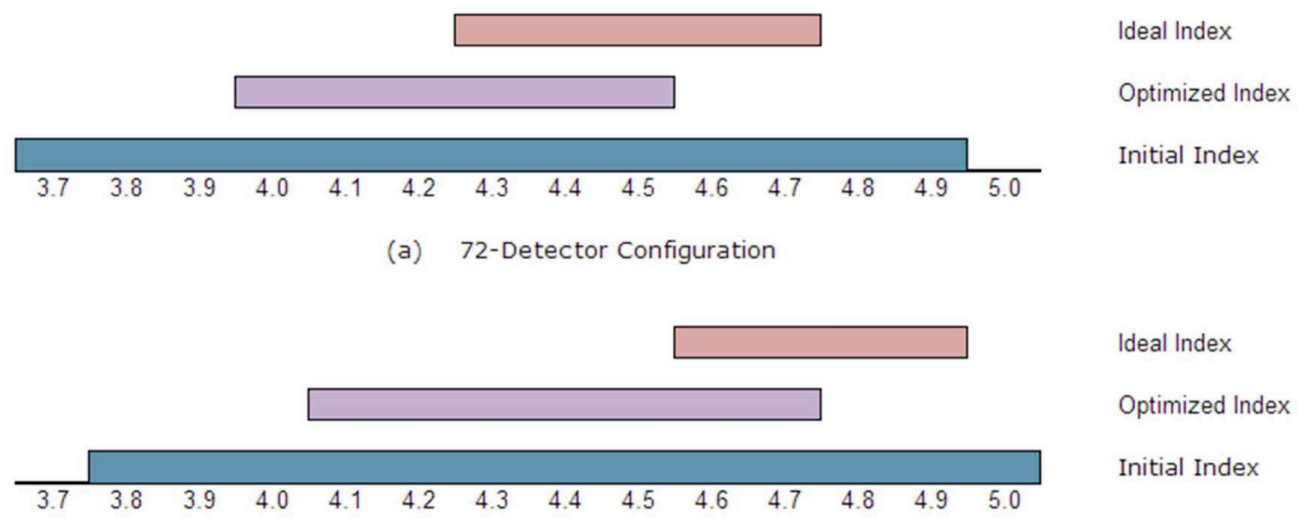

(b) 76-Detector Configuration

Fig. 3. Changes in the Index of Distribution.

Table 2. Summary of Detector Distribution for 72-Detector Configuration.

\begin{tabular}{c|c|c|c|c|c|c}
\hline \multirow{3}{*}{ Design } & \multirow{2}{*}{$\begin{array}{c}\text { Safety } \\
\text { Channel }\end{array}$} & \multicolumn{4}{|c|}{ Quadrant } & \multirow{2}{*}{$\boldsymbol{I}_{\text {Dist }}$} \\
\cline { 2 - 6 } & & $\mathbf{1}$ & $\mathbf{2}$ & $\mathbf{3}$ & $\mathbf{4}$ & \\
\hline \multirow{4}{*}{ Initial } & $\mathrm{A}$ & 4 & 6 & 4 & 4 & 4.4 \\
\cline { 2 - 7 } & $\mathrm{B}$ & 7 & 5 & 4 & 2 & 3.7 \\
\cline { 2 - 7 } & $\mathrm{C}$ & 5 & 5 & 4 & 4 & 4.3 \\
\cline { 2 - 7 } & $\mathrm{D}$ & 4 & 3 & 5 & 6 & 4.9 \\
\hline \multirow{5}{*}{ Optimized } & $\mathrm{A}$ & 5 & 6 & 5 & 2 & 4.0 \\
\cline { 2 - 7 } & $\mathrm{B}$ & 5 & 4 & 4 & 5 & 4.5 \\
\cline { 2 - 6 } & $\mathrm{C}$ & 6 & 4 & 3 & 5 & 4.3 \\
\cline { 2 - 6 } & $\mathrm{D}$ & 4 & 5 & 5 & 4 & 4.5 \\
\hline
\end{tabular}

Table 3. Summary of Detector Distribution for 76-Detector Configuration.

\begin{tabular}{|c|c|c|c|c|c|c|}
\hline \multirow{2}{*}{ Design } & \multirow{2}{*}{$\begin{array}{c}\text { Safety } \\
\text { Channel }\end{array}$} & \multicolumn{4}{|c|}{ Quadrant } & \multirow{2}{*}{$\boldsymbol{I}_{\text {Dist }}$} \\
\hline & & 1 & 2 & 3 & 4 & \\
\hline \multirow{4}{*}{ Initial } & A & 5 & 3 & 5 & 6 & 5.0 \\
\hline & B & 5 & 5 & 7 & 2 & 4.4 \\
\hline & $\mathrm{C}$ & 8 & 5 & 4 & 2 & 3.8 \\
\hline & $\mathrm{D}$ & 7 & 5 & 4 & 3 & 4.1 \\
\hline \multirow{4}{*}{ Optimized } & A & 6 & 6 & 5 & 2 & 4.1 \\
\hline & B & 7 & 4 & 4 & 4 & 4.3 \\
\hline & $\mathrm{C}$ & 6 & 5 & 6 & 2 & 4.2 \\
\hline & $\mathrm{D}$ & 6 & 3 & 5 & 5 & 4.7 \\
\hline
\end{tabular}

have been somewhat optimized during this process. Therefore, the incremental improvement to the TSP value around $0.50 \% \mathrm{FP}$ is not totally unexpected.

\section{CONCLUSIONS}

It has been demonstrated in this paper that additional optimization related to how the ROP detectors are placed into the safety channels can indeed provide additional improvement in the trip set point value. The methodology used for the channelization optimization has been discussed in detail. Some examples from numerical experiments using various sizes of ROP system have been presented. It has been shown that a gain of up to $0.56 \% \mathrm{FP}$ can be realized by performing the channelization optimization for a 4-channel system. Finally, it should also be noted that this methodology is also applicable to the existing CANDU reactors and could be used as an option for addressing the ROP-related ageing issue.

\section{NOMENCLATURES}

\section{CANDU CANada Deuterium Uranium \\ CCP Critical Channel Power \\ CP Channel Power}

DETPLASA DETector PLAcement using Simulated

MTD Margin-To-Dryout

MTT Margin-To-Trip

NOP Neutron Overpower Protection

OID Onset of Intermittent Dryout

ROP Regional Overpower Protection

SDS Shutdown System

SIR Straight Individually Replaceable

SOR Shutoff Rod

SLOR Slow Loss Of Regulation

TSP

Trip Set Point 


\section{REFERENCES}

[1] M. Levine, L. Blake, E. Attia, "SIMBRASS and Neutron Overpower Analysis," Proc. $22^{\text {nd }}$ Nuclear Simulation Symposium, Ottawa, Canada, November 2002.

[2] A. H. Chung, M. Khanna, "SIMBRASS: a Monte-Carlo Model for the Assessment of the Unavailability of Regional Overpower Trip Systems," Proc. First Annual CNS Conference, Montreal, Canada, June 1980.

[ 3 ] C. M. Bailey and F. A. R. Laratta, "Design and Assessment of the Neutron Overpower Trips in the 600 MWe CANDU-
PHW Reactors," TDAI-315, Atomic Energy of Canada Limited, Mississauga, ON, Canada, 1982.

[4] D. Kastanya, V. Caxaj, "Methodologies for Optimizing ROP Detector Layout for CANDU ${ }^{\circledR}$ Reactors," Annals of Nuclear Energy, 38, No.1, pp. 21-31, 2011.

[5] S. Kirkpatrik, C. Gelatt, M. Vecchi, "Optimization by Simulated Annealing," Science, 220, 671-680, 1983.

[6] D. Kastanya, V. Caxaj, "Regional Overpower Protection Analysis for CANDU 6 Reactors using ROVER-F Code," Annals of Nuclear Energy, 37, No.1, pp. 28-33, 2010. 